\title{
Customer Satisfaction at Galen House, Bulawayo, Zimbabwe, 2019
}

\author{
Munekayi Padingani ${ }^{1,2^{*}}$, Nothando Dube ${ }^{2}$, Simbarashe Chiwanda ${ }^{1}$, Notion Gombe ${ }^{1}$, \\ Gerald Shambira ${ }^{1}$, Ms T. Juru1, Peter Nsubuga1, Mufuta Tshimanga1 \\ ${ }^{1}$ Department of Community medicine, University of Zimbabwe, Harare, Zimbabwe \\ ${ }^{2}$ Galen House 24 Hours Medical Practice, Bulawayo City, Zimbabwe \\ Email: ^drmunepadi@gmail.com, ^munepadi@yahoo.fr, noencane@gmail.com,chiwandas@gmail.com, \\ tshimangamufuta@gmail.com,gshambira@yahoo.com,gombent@yahoo.com,pnsubuga@globalphsolutions.com, \\ tsitsijuru@gmail.com
}

How to cite this paper: Padingani, M., Dube, N., Chiwanda, S., Gombe, N., Shambira, G., Juru, M.T., Nsubuga, P. and Tshimanga, M. (2021) Customer Satisfaction at Galen House, Bulawayo, Zimbabwe, 2019. Open Journal of Epidemiology, 11, 70-79. https://doi.org/10.4236/ojepi.2021.111007

Received: December 10, 2020

Accepted: February 1, 2021

Published: February 4, 2021

Copyright $\odot 2021$ by author(s) and Scientific Research Publishing Inc. This work is licensed under the Creative Commons Attribution International License (CC BY 4.0).

http://creativecommons.org/licenses/by/4.0/

\begin{abstract}
Introduction: Healthcare industries have seen recent movements towards continuous quality improvement. The healthcare regulators shifted towards a market-driven approach of turning patient satisfaction surveys into a quality improvement tool for overall organizational performance. Data concerning this has remained limited in private sector in Zimbabwe. Thus, this study aims to determine patients and health workers perception about health services offered at Galen house in order to enable the institution to come up with evidence-based interventions to improve the quality of healthcare services. Methods: This was a descriptive cross sectional study. It was a mixed qualitative and quantitative study involving data collected from patients/ guardians and health workers at Galen House from January 2019 to March of the same year. Data was analysed using Microsoft Excel version 2013. Results: There were a total of 270 patients involved into the study. 173 (64\%) perceived the waiting before services as good. $213(79 \%)$ perceived the total waiting time as not long. Privacy 215 (80\%) and confidentiality 223 (83\%) perceived as good by patients. More than $50 \%$ of health workers perceived health services offered at Galen House as good. Staff attitude was perceived as good $191(71 \%)$ by patients in contrary to some instance by health workers. Affordability of services was perceived as manageable. Conclusion: The total waiting time was perceived as not long at Galen House. Staff attitude was perceived differently by patients and health workers. Improving those two will contribute to more utilization of services by the community.
\end{abstract}

\section{Keywords}

Patient Satisfactory Survey, Private Sector, Patient Waiting Time, Patient 
Privacy, Patient Confidentiality, Patient Affordability

\section{Introduction}

Healthcare industries have seen recent movements towards continuous quality improvement [1]. Avedis Donabedian, a physician and health services researcher, developed the original model of quality of health services by incorporating patient perception into quality assessment [2]. The model gives information about quality of care drawn from three categories: structure, process and outcomes. Structure describes where the care is given, taking into consideration, buildings, staff, financing and equipment. Process is about interactions between patients and providers during the delivery of healthcare. Outcomes consider as effects of healthcare on the health status of patients and populations [3].

In 1985, Notre Dame professors Irwin Press, a medical anthropologist, and Rod Ganey, a sociologist and statistician created a new market. They brought the science of survey design and administration to healthcare. Press and Ganey started out with just a handful of hospital clients. Over time, more hospitals saw the value that could be gained from tracking their patients' satisfaction [4].

Nowadays, more healthcare organizations, including clinics, and General practitioners rooms are conducting patient satisfaction surveys [5].

The healthcare managers that are passionate about excellence take patient perception into account to come up with strategies to improve quality of care in their institutions. The healthcare regulators shifted towards a market-driven approach of turning patient satisfaction surveys into a quality improvement tool for overall organizational performance [6].

Zimbabwe assumed the Primary Health Care approach in 1980 and its health system is structured accordingly. The health services delivery platforms include primary (clinic), secondary (district hospital), tertiary (provincial hospital) and quaternary (central hospital) facilities [7]. Ninety percent of these health facilities are at primary care level which refers complicated cases to the next levels of care [8]. Mission and private facilities provide considerable services mostly in rural and urban areas respectively [7].

Quality of care in health institutions is a challenge worldwide. To continuously improve this, the patient's satisfactory survey approach has been used for years. A study done in Wuhan public hospitals in China showed that patients were satisfied with services received [9]. Nwaeze in Nigeria found that $81 \%$ of participants perceived the services received as good [10].

The parliament of Zimbabwe commissioned Customer Satisfaction Survey in 2016 as part of a tracking mechanism on how customers, both internal and external view the services that they receive. Despite the importance of patients' satisfactory survey, there is currently no evidence of large scale use of the survey in Zimbabwe. Patients Satisfactory Survey has been limited to few public institutions, with patient waiting time of less than 2 hours in Tsholotsho district Hos- 
pital [11]. There is no evidence on the use of Patients Satisfaction Survey in private sector in Zimbabwe. It is recommended to have frequent Patients Satisfaction Survey in order to improve the quality of services offered to patients and or clients. Against this background, we aimed to investigate patients and health workers perception about health services offered at Galen house; a private facility and make recommendations for the improvement of quality of services offered to the communities.

\section{Methods}

\subsection{Study Design}

We conducted a descriptive cross sectional study to investigate patients and health workers perception about health services offered at Galen house.

\subsection{Study Setting}

Zimbabwe is a landlocked country in south-central Africa. It is bordered by Botswana on the west, Zambia on the north, Mozambique on the east, and South Africa on the south. The country is divided into 10 provinces and 59 districts with a population of 13,061,239 according to 2012 Census projection [12]. The country has a life expectancy of 61 years largely driven by deaths due to infectious diseases (2). Access to quality health care is a major challenge in areas outside the two major cities of Harare and Bulawayo.

The study was conducted at Galen house in Bulawayo, second biggest urbanized city. Galen house a 14-bed admitting private medical facility which offers 24 hours emergency services to everyone regardless of socio-economic status. The institution provides preventive, promotive, curative and rehabilitation services.

\subsection{Study Population}

All patients ( 15 years old and above patients or clients and guardians of below the same age group) attending Galen House in the City of Bulawayo from January to March 2019. Available health workers of different departments.

Inclusion criteria: All consenting patients and or guardians attending Galen house in Bulawayo from January to March 2019. All consenting available health workers during the same period.

Exclusion criteria: Patients and or guardians not consenting, emergencies, very ill patients and clients not attending Galen house in Bulawayo. Non consenting available health workers.

\subsection{Data Collection}

We conducted three focus group discussions with key informants available on the day of the session to know about perception of quality of care rendered to patients. Key informants were staff from different departments. First group made of clerks, second with nurses and the last with nurse aids and porters together. 
We used an interviewer administered questionnaire to collect data from patients and or guardians attending Galen house, to elicit information on socio-demographic and socio-economic characteristics and patient's perception about services received.

\subsection{Data Management and Analysis}

We checked for data quality using completeness of data entries in the questionnaire. We analysed using Microsoft Excel version 2013 to generate frequencies, medians and proportions.

\subsection{Permission and Ethical Consideration}

Permission to carry out the study was sought and obtained from Galen house administrator.

Informed written consent was obtained from study participants. Patients' names were not used in the questionnaire. We kept the data safe and confidential and only used it for this study.

\subsection{Sampling}

We used Dobson formula to calculate sample size and the parameters from IL Nwaeze in a study done in Nigeria the proportion of participants perceiving the services received as good was $81 \%$ [10]. Using results from the above study and accepting a precision of $5 \%$ and $95 \%$ confidence interval $(Z=1.96)$, a minimum of 236 study participants was required but we interviewed 270 participants. Dobson formula $n=Z^{2} p q / \Delta^{2}$.

Sampling design: All patients and or guardians attending Galen House from January to March 2019 responding to the inclusion criteria were enrolled into the study.

\section{Results}

\subsection{Characteristics of FGD Participants/Health Workers}

A total of three focus group discussions were conducted with fifteen staff on duty. The first one with clerks (accounts department), second one with nurses and the last one with porters and nurse aids together. The majority of health workers were females $(87 \%, 2 / 13)$; their overall median age was 46 years and median years in service was 10 years (Table 1 ).

Table 1. Characteristics of focus group discussion participants.

\begin{tabular}{ccccc}
\hline Characteristic & \multicolumn{3}{c}{ Department } \\
Gender & Nursing & Account & General hand/Porters & Total \\
\hline Male & 0 & 0 & 2 & 2 \\
Female & 8 & 5 & 0 & 13 \\
Median age in years & 45.5 & 42 & 53.5 & 13.5 \\
Median years in service & 8 & 11 &
\end{tabular}




\subsection{Health Worker Perceived Quality of Services}

Health workers reported the policy of first arrived and first served is in place at Galen House with exception to emergency cases. Patient waiting time, privacy and confidentiality were perceived by more than $50 \%$ of health workers as good and can be made excellent with less effort. Attitude of staff was perceived as poor in some instance by health workers. Total service time was perceived as average. Affordability of services was perceived as manageable in view of the current economic situation.

\subsection{Demographic and Socio-Economic Characteristics of Patients/Guardians}

We enrolled 270 patients and or guardians attending Galen House into the study. The median age of patients was 38 years with a range of 30 to 49 years. $86 \%$ $(232 / 270)$ of study participants stay in urban area, $55 \%$ went to school up to tertiary level, $70 \%$ are married and $38 \%$ are formally employed (Table 2).

Table 2. Socio-demographic and socio-economic characteristics of patients/guardians attending Galen House, Jan to March 2019.

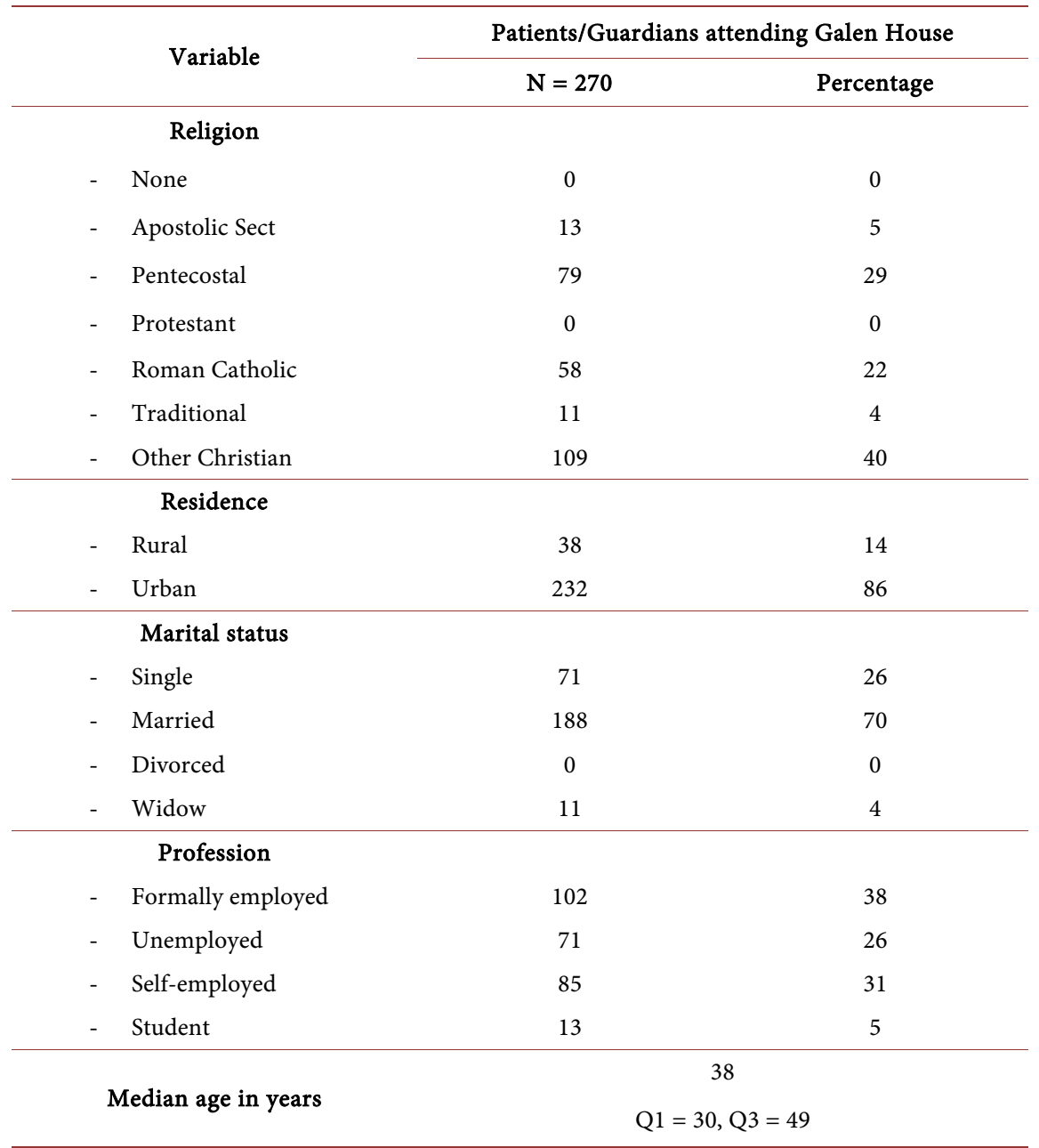

Age: $\operatorname{Min}=16, \operatorname{Max}=75$. 


\subsection{Patients/Guardians Perception of Services at Galen House, Jan to March 2019}

Sixty four percent (173/270) of patients/guardians perceived the waiting time before services at Galen House as good (Figure 1). Eighty percent (215/270) of patients/guardians perceived privacy and confidentiality at Galen House as good (Figure 2). Seventy one percent (191/270) perceived Galen House staff attitude as good (Figure 3) and eighty four percent (227/270) perceived the cost of services as manageable. Seventy nine percentage (213/270) of patients/guardians perceived as Good the total time spent at Galen House (Figure 4).

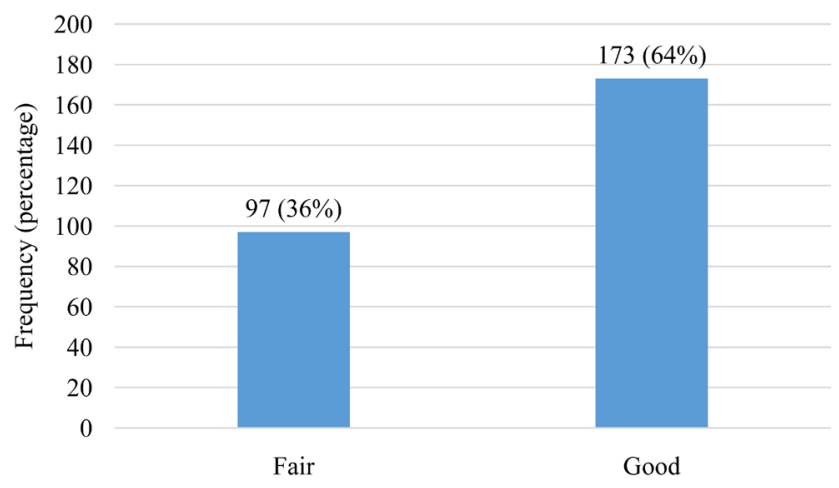

Figure 1. Patients/guardians perceived waiting time before services at Galen House, January to March 2019.

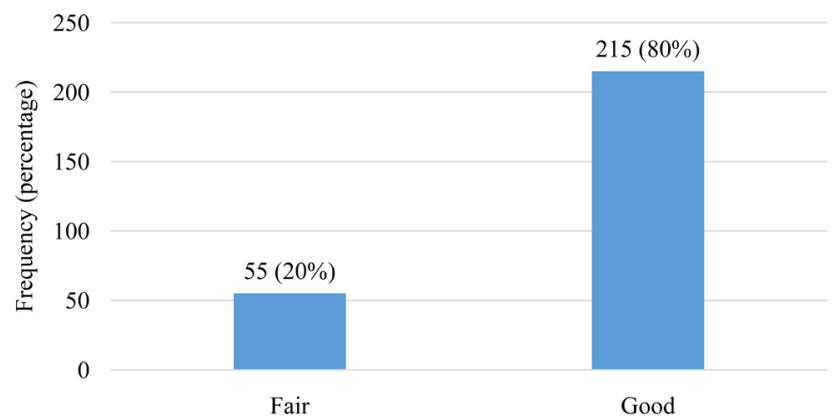

Figure 2. Patients/guardians perceived privacy and confidentiality at Galen House, January to March 2019.

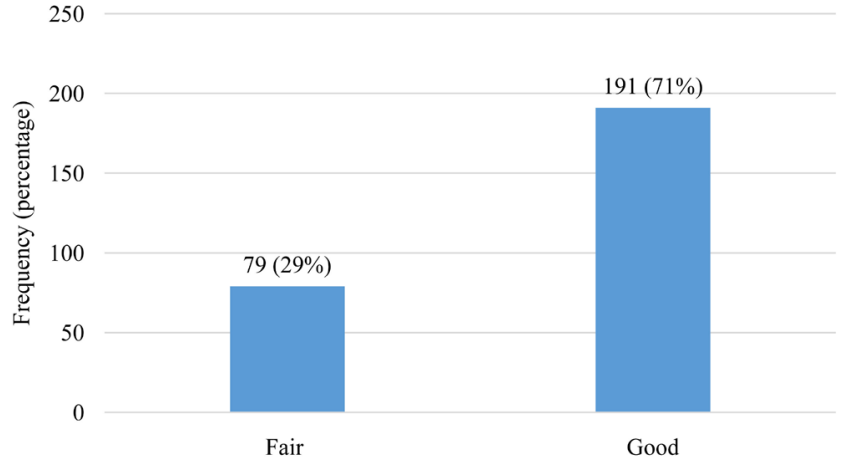

Figure 3. Patients/guardians perceived staff attitude toward patients at Galen House, January to March 2019. 


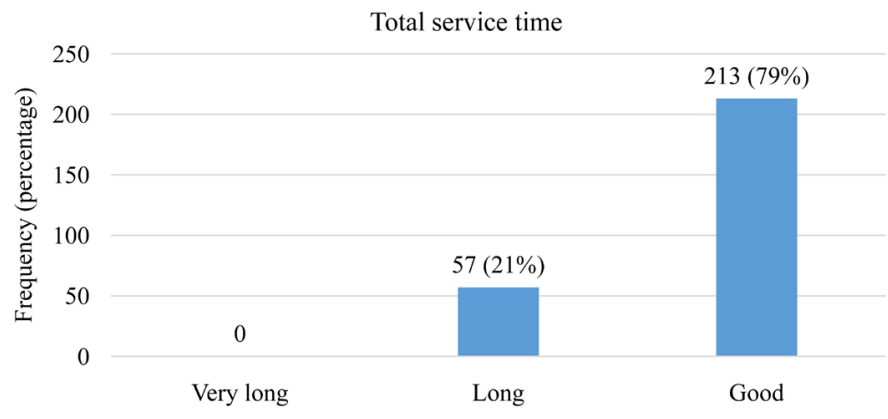

Figure 4. Patients/guardians perceived total service time at Galen House, January to March 2019.

\section{Discussion}

Our study revealed that triage of patients is in use at Galen house. Patients are assessed on arrival if critical, they are given priority meaning they get to be served first. Patient waiting time is an important parameter in the assessment of quality of care and patients' satisfaction towards healthcare services [13]. This affects utilization of health services, Patients perceive long waiting times as a barrier to actually obtaining services [14]. In our study the waiting time before services was perceived as good by two third of patients and the total waiting time from reception to the administration of stat dose medicine was as well perceived as good. This is similar to findings by Tran TD in Vietnam where the average waiting times from registration to preliminary diagnosis in 2014 was $50.41 \mathrm{mi}-$ nutes, and in 2015 was 42.05 minutes [13]. This patient's perception may be explained by long time spent at public health facilities when seeking services. In Zimbabwe public health facilities, two hours or less is the acceptable patient's waiting time.

Patient's right to privacy involves the confidentiality of information related to the patient and bodily privacy of the patient [15]. Maintaining patients' privacy and confidentiality has always been an ethical obligation of health workers [16]. Patients perceived privacy and confidentiality at Galen House as good. This may be attributed to health workers knowledge of patients' right. This is supported by Nilufer Demirsoy who said the purpose of patient rights as a basic component of human rights, is to support patients and to strengthen them in social terms [17].

The health worker attitudes and behaviours towards clients/patients influence the use of these services. Patients perceived health workers attitude at Galen House as good and the majority of Galen House staff is made of female. Females show more compassion and empathy towards others. This is supported by Caterina Ledda at $\mathrm{Al}$ study in which female health workers showed more acceptance towards drug users than male health workers [18]. This is contrary to Galen house health workers perception of their own attitude which they rated poor. This may be attributed to low motivation due to the country economic situation.

Primary health care is defined as essential healthcare based on practical, scientifically sound and socially acceptable methods and technology, made universally accessible to the communities through their full participation and at a cost that 
is affordable and can be maintained at every stage of the communities' development [19]. Primary health care became a core policy for WHO in 1978, with the adoption of the Declaration of Alma-Ata and the strategy of "Health for all by the year 2000" [20]. Our study findings on affordability of services was contrary to the primary health care approach because some patients perceived the services as expensive due the current economic situation prevailing in the country.

Quality of services provided at Galen House was regarded as good by more than $60 \%$ of respondents which is similar to a study done in Idadan Nigeria where $55 \%$ of study participants perceived quality of services as good [21]. This could be explained by the degrading health service deliveries at public health facilities in the country due to several health workers (nurses and doctors) strikes.

\section{Study Limitations}

Though this study was undertaken quite rigorously, however, it was not without limitations. Patients or patients' guardians were interviewed and self-reported information was collected, there is a possibility of under and over reporting. Recall bias is a possibility in this study. Generalization of findings can be an issue, as to what extent the services offered at Galen House is representative of the private health services in Bulawayo or Zimbabwe.

\section{Conclusions}

The patient waiting time before services and patient total waiting time (from reception to administration of stat dose medicine) at Galen House was perceived as good by patients and health workers. Galen House staff attitude was perceived as good by patients, but perceived as poor by health workers in some instance. Quality of services provided at Galen House was regarded as good by more than $60 \%$ of respondents. Staff attitude could be improved by increasing staff remuneration, organising training and refresher courses for health workers and the use of disciplinary procedures.

\section{What is already known on this topic?}

Systematic reviews show that quality of services was perceived as good in many studies.

While there are studies assessing quality of services among patients in public sector, there is no evidence from private sector from Zimbabwe.

\section{What this study adds?}

Galen House a private health institution in Bulawayo Zimbabwe, where we found that $64 \%$ of patients/guardians perceived patient waiting time as good. Staff attitude was perceived as poor in certain instance by health workers

\section{Acknowledgements}

We thank Galen House partners especially Dr M. Fallala and Dr N. Ndebele, Galen house staff and patients/guardians who consented to participate in the study for making this research possible. 


\section{Conflicts of Interest}

The authors declare no competing interest.

\section{Authors' Contributions}

All authors were responsible for the conception of the problem, design, collection, analysis and interpretation of data and drafting the final article. The manuscript was read and approved by all authors.

\section{References}

[1] Dawn, A.G. and Lee, P.P. (2003) Patient Satisfaction Instruments used at Academic Medical Centers: Results of a Survey. The American Journal of Medical Quality, 18, 265-269. https://doi.org/10.1177/106286060301800607

[2] McDonald, D.K., Kathryn, M., et al. (2007) Closing the Quality Gap: A Critical Analysis of Quality Improvement Strategies. Vol. 7. Care Coordination. Agency for Healthcare Research and Quality (US), Rockville.

[3] Donabedian, A. (1988) The Quality of Care. How Can It Be Assessed? JAMA, 260, 1743-1748. https://doi.org/10.1001/jama.1988.03410120089033

[4] Siegrist Jr., R.B. (2013) Patient Satisfaction: History, Myths, and Misperceptions. AMA Journal of Ethics, 15, 982-987. https://doi.org/10.1001/virtualmentor.2013.15.11.mhst1-1311

[5] Schlesinger, V. (2019) Importance of Conducting Patient Satisfaction Surveys. https://blog.smartsurvey.co.uk/importance-of-conducting-patient-satisfaction-surv eys

[6] Al-Abri, R. and Al-Balushi, A. (2014) Patient Satisfaction Survey as a Tool towards Quality Improvement. Oman Medical Journal, 29, 3-7. https://doi.org/10.5001/omj.2014.02

[7] Ministry of Health and Child Care Zimbabwe (2016) National Health Strategy for Zimbabwe 2016-2020. Harare.

[8] Zimbabwe Ministry of Health and Child Care (2019) Zimbabwe District Health Information Software 2012. Harare.

[9] Meng, R.T., Li, J.J., Zhang, Y.Q., Yu, Y., Luo, Y., et al. (2018) Evaluation of Patient and Medical Staff Satisfaction Regarding Healthcare Services in Wuhan Public Hospitals. International Journal of Environmental Research and Public Health, 15, 769. https://doi.org/10.3390/ijerph15040769

[10] Nwaeze, I.L., Enabor, O.O. and Oluwasola, T.A.O. (2013) Perception and Satisfaction with Quality of Antenatal Care Services among Pregnant Women at the University College Hospital, Ibadan, Nigeria. Annals of Ibadan Postgraduate Medicine, 11, 22-28.

[11] Tsholotsho District Health Executive (2019) Patient Satisfaction Survey, Tsholotsho District Report.

[12] Zimbabwe National Statistics Agency (2012) Zimbabwe Population Census 2012 National Report.

http://www.zimstat.co.zw/wp-content/uploads/publications/Population/population/ census-2012-national-report.pdf

[13] Tran, T. (2017) Patient Waiting Time in the Outpatient Clinic at a Central Surgical Hospital of Vietnam: Implications for Resource Allocation. F1000Research, 6, 454. https://doi.org/10.12688/f1000research.11045.1 
[14] Oche, M. (2013) Determinants of Patients Waiting Time in the General Outpatient Department of a Tertiary Health Institution in North Western Nigeria. Annals of Medical and Health Science Research, 3, 588-592.

https://doi.org/10.4103/2141-9248.122123

[15] Ocaktan, E., Yıldız, A. and Özdemir, O. (2004) Knowledge and Attitude of Health Care Personnel Working at Abidinpasa Health Group Directorate. Ankara Üniversitesi Tip Fakültesi, 57, 129-137.

[16] McCullough, J. (2013) Maintaining Patients' Privacy and Confidentiality with Family Communications in the Intensive Care Unit. Critical Care Nurse, 33, 77-79. https://doi.org/10.4037/ccn2013310

[17] Demirsoy, N. and Kirimlioglu, N. (2016) Protection of Privacy and Confidentiality as a Patient Right: Physicians' and Nurses' Viewpoints. BioMed Research, 27, 1437-1448.

[18] Ledda, C., et al. (2017) Attitude of Health Care Workers (HCWs) toward Patients Affected by HIV/AIDS and Drug Users: A Cross-Sectional Study. International Journal of Environmental Research and Public Health, 14, 284. https://doi.org/10.3390/ijerph14030284

[19] ACT Academy (2019) A Model for Measuring Quality Care. ACT Academy. https://improvement.nhs.uk/documents/2135/measuring-quality-care-model.pdf

[20] World Health Organization (1978) Declaration of Alma-Ata. WHO, Geneva.

[21] Falayi, E.O., et al. (2018) Patients' Perception of Quality of Diabetes Care Received in Ibadan, Nigeria. Archives of Basic and Applied Medicine, 6, 119-125. 